\title{
Diffusion in a generalized Rubinstein-Duke model of electrophoresis with kinematic disorder
}

\author{
Richard D. Willmann* and Gunter M. Schütz \\ Institut für Festkörperforschung, Forschungszentrum Jülich, 52425 Jülich, Germany \\ Kavita Jain \\ Department of Theoretical Physics, Tata Institute of Fundamental Research, Homi Bhabha Road, Mumbai 400 005, India
}

(Received 20 January 2003; published 30 June 2003)

\begin{abstract}
Using a generalized Rubinstein-Duke model, we prove rigorously that kinematic disorder leaves the prediction of the standard reptation theory for the scaling of the diffusion constant in the limit for long polymer chains $D \propto L^{-2}$ unaffected. Based on an analytical calculation as well as on Monte Carlo simulations, we predict kinematic disorder to affect the center-of-mass diffusion constant of an entangled polymer in the limit for long chains by the same factor as single particle diffusion in a random barrier model.
\end{abstract}

DOI: 10.1103/PhysRevE.67.061806

PACS number(s): $61.25 . \mathrm{Hq}, 83.10 . \mathrm{Kn}$

\section{INTRODUCTION}

The derivation of large scale properties of polymer systems, such as viscosity and diffusion constant out of microscopic models, is among the basic problems of polymer science. For entangled polymers in gels or melts, de Gennes [1] predicted scaling laws for the dependence on the polymer length $L$ for viscosity $\left(\eta \propto L^{3}\right)$ and diffusion constant ( $D$ $\left.\propto L^{-2}\right)$. These scaling laws are assumed to be valid for the limiting case of polymer length going to infinity. In experiments, the apparent scaling laws $D \propto L^{-2.4}$ and $\eta \propto L^{3.4}$ are found $[2-4]$. However, the experimental findings do not contradict the predictions of reptation, as a crossover due to decreasing finite size effects with increasing chain length cannot be ruled out [3]. Presumably, contour length fluctuations (CLF) are one of the causes for the deviating scaling exponent for finite chain length [5-7]. In the framework of the repton model introduced by Rubinstein [8,9] and Duke $[10,11]$ (further on to be called RD model), which incorporates CLF, it is possible to calculate viscosity, diffusion coefficient, and other quantities of interest. A good agreement with both theoretical and experimental results is found $[6,7,12,13]$. Using the RD model with periodic boundary conditions, Kooiman and van Leeuwen [14] analytically calculated the proportionality constant $c$ for the diffusion constant in the limit for infinite chain length: $\lim _{L \rightarrow \infty} D L^{2}=c$. They found $c=w /(2 d+1), d$ being the dimensionality of the entanglement network and $w$ a model constant defining the time scale. Building upon this result, Prähofer and Spohn [15] rigorously derived the leading order term in $1 / L$ for the diffusion constant in the RD model and furthermore proposed a scaling for the finite size effects: $D L^{2}-W /(2 d$ $+1) \propto L^{-\beta}$, where $1 / 2 \leqslant \beta \leqslant 1$. All results mentioned above were obtained under the assumption that the entanglement network which is topologically restricting the polymer is regular and static. Real entanglement networks such as gels or melts are disordered. The effects of a disordered environment can be manifold: (1) Spatial variations of the mobility of the "defects" of stored length; (2) locally fluctuating po-

*Electronic address: r.willmann@fz-juelich.de tential energy due to interactions between chain and environment; (3) entropically favorable regions of low entanglement density; (4) relaxation of the environment (constraint release).

Numerical investigations [16] showed that entropically favorable regions can for short chains substantially lower the diffusion constant by the creation of "entropic traps." So far, conclusive investigations of diffusing polymers, long enough to span several such traps, are missing. Constraint release is considered to be of minor importance in gels but needs to be self-consistently taken into account in melts [17]. Schäfer, Baumgärtner, and Ebert [18] numerically investigated the effect of kinematic disorder, i.e., disorder which affects the mobility of the chain segments while leaving the equilibrium distribution of chain configurations unaltered. Their investigation shows that the reptation prevails in presence of kinematic disorder. However, due to being based on Monte Carlo (MC) simulations and thus relatively short chains, no quantitative result could be obtained about the modifications the disorder would cause to the reptation prediction for the diffusion constant in the long chain limit. As reptation is shown to prevail, the scaling $\lim _{L \rightarrow \infty} D L^{2}=c$ must remain valid. The aim of this paper is to calculate the coefficient $c$ for a polymer diffusing in a disordered environment exhibiting kinematic disorder. This means that we are interested in the behavior of the chain in the long chain length limit originally envisaged by the standard reptation theory. Some of the results presented here in detail were briefly reported in an earlier paper [19].

The paper is divided into six sections: In Sec. I, the definition of the model is given and the master equation for the chain dynamics is presented in terms of the quantum Hamiltonian formalism [20]. This master equation yields the stationary distribution of chain conformations as shown in Sec. II. The model with periodic boundary conditions is analyzed in Sec. III. Adapting the strategy in Ref. [14] for obtaining the diffusion constant for the periodic system yields a lower bound on the diffusion constant for the original system. Section IV is devoted to deriving an upper bound to the diffusion constant by generalizing a variational approach used in Ref. [15] for the ordered system. In Sec. V, Monte Carlo simula- 

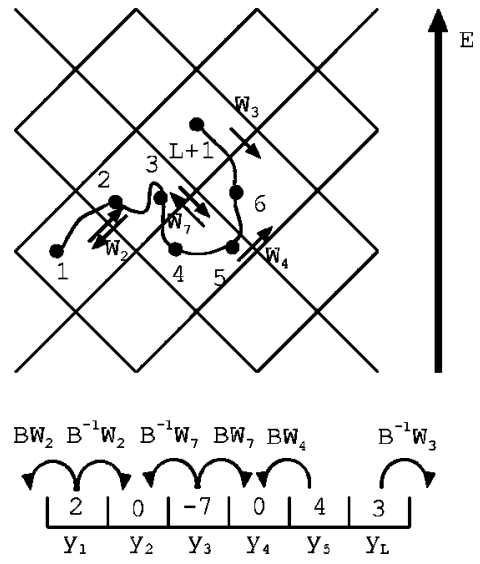

FIG. 1. Two-dimensional representation of a kinematically disordered lattice with a reptating polymer chain and the lattice gas mapping. Arrows indicate possible moves of the reptons. $B$ $=\exp (E / 2)$.

tions of the polymer diffusion in kinematically disordered environments are presented. The last section addresses the dynamics of internal chain segments.

\section{A LATTICE MODEL FOR REPTATION WITH KINEMATIC DISORDER}

The RD model is a discretized model for reptation, i.e., it describes the dynamics of a polymer in an entanglement network. This network is assumed to be regular and static. In three dimensions, it has the shape of a cubic lattice, where the polymer is forbidden to cut through the edges of the cubes. The faces of the cubes can by penetrated by the chain (see Fig. 1). The polymer itself is assumed to consist of $L$ +1 "reptons," i.e., segments of about the entanglement length, which equals the lattice constant of the cubic lattice.

The model defines dynamical rules for the reptons' motion. The dynamics on a smaller scale, i.e., high frequency Rouse modes, is averaged out. In this sense, the RD model is an effective model. The dynamical rules for the reptons are the following.

(a) Each cell occupied by the chain must contain at least one repton to ensure the connectivity of the chain, since the length represented by a repton equals the lattice constant of the cells. The sequence of occupied pores corresponds to the tube as in the standard reptation theory.

(b) End reptons can move to adjacent cells provided rule (a) is not violated.

(c) Interior reptons can move to cells occupied by the neighboring reptons if allowed by rule (a). This ensures the dynamics to be reptation, as any movement in the interior requires multiple reptons to occupy the same cell, which means that there is some excess "stored length" available. This corresponds to the motion of defects in the original de Gennes reptation model.

All moves are assumed to be thermally activated. In our model, the cubic lattice representing the entanglement network gets kinematically disordered by assigning an individual energy barrier to every boundary between adjacent cells. These barriers have to be overcome by a crossing rep- ton. Note that we do not assign different energies to the reptons when residing in the cells. This choice of quenched disorder guarantees that only the mobility of the reptons, but not the equilibrium configurations, is affected, as will be seen below. Let there be $\sigma \in N$ different possible hopping rates $W_{\alpha}$, which are distributed randomly and occur with a probability $f\left(W_{\alpha}\right)$ throughout the lattice. We demand that the disorder averages $\langle 1 / W\rangle=\sum_{\alpha=1}^{\sigma} f\left(W_{\alpha}\right) / W_{\alpha}$ and $\left\langle 1 / W^{2}\right\rangle$ (the mean hopping time and its second moment) are finite.

The RD model is used to describe the dynamics of an entangled polymer chain under the influence of an external electric field. A common example is DNA under electrophoresis conditions where the reptons carry a charge each and develop a drift velocity along the direction of an applied electric field. Let the field be oriented along the (111) diagonal of the cubes. We denote the (dimensionless) energy gain of a repton when moving from one cell to another along the direction of the field as $E$. By local detailed balance, moves across a cell boundary with assigned hopping rate $W_{\alpha}$ along the field happen with rate $W_{\alpha} \exp (E / 2)$, those in the opposite direction with rate $W_{\alpha} \exp (-E / 2)$ [10]. By projecting the reptons' positions on the direction of the field, their relative coordinates can be denoted as a one-dimensional lattice gas with $L$ sites by the following prescription.

(1) If the projected link between adjacent reptons $i$ and $i$ +1 is oriented along (against) the field direction across a cell boundary with assigned rate $W_{\alpha}$, site $i$ of the lattice gas is assigned the value $y_{i}=\alpha\left(y_{i}=-\alpha\right)$. We interpret values $\pm \alpha$ as particles.

(2) If adjacent reptons $i$ and $i+1$ occupy the same cell, i.e., their projected positions coincide, the link is represented in the lattice gas as $y_{i}=0$ at site $i$. We interpret a site, which is assigned a 0 as being unoccupied.

Thus, the $L+1$ coordinates of the reptons in the direction of the field are translated into the equivalent set of the relative coordinates manifest as the assignment of $\alpha,-\alpha$, or 0 to the $L$ sites of the lattice gas plus the center of mass coordinate's component in field direction. The dynamics of the reptons translates into the lattice gas picture as follows. In the bulk, particles of sort $\pm \alpha$ hop to the left with rate $W_{\alpha} \exp ( \pm E / 2)$ and to the right with rate $W_{\alpha} \exp (\mp E / 2)$, where each site can be occupied by at most one particle. The end dynamics in the lattice gas picture needs some care. Assuming $y_{1}\left(y_{L}\right)$ to be nonzero, the only possible move is the retraction of the end repton to the cell occupied by it's neighbor [rule (a)]. This retraction, being a particle annihilation event in the lattice gas picture, happens with the same rate as the respective move in the bulk. Assuming $y_{1}\left(y_{L}\right)$ to be zero, the end repton can, according to rule (b), move to any of the $2 d$ adjacent cells. For half of these, the move leads to links being along the field direction, the other half against it. The probability of the chosen move leading to the crossing of a cell boundary with rate $W_{\alpha}$ being assigned to it is $f\left(W_{\alpha}\right)$. Thus, the move of the repton, being a particle creation event in the lattice gas picture, leads to $y_{1}\left(y_{L}\right)$ changing from 0 to $\pm \alpha$ with rate $\exp (\mp E / 2) f\left(W_{\alpha}\right) W_{\alpha} d\left[\exp ( \pm E / 2) f\left(W_{\alpha}\right) W_{\alpha} d\right]$. This choice of boundary dynamics is, on an average, correct, but neglects 


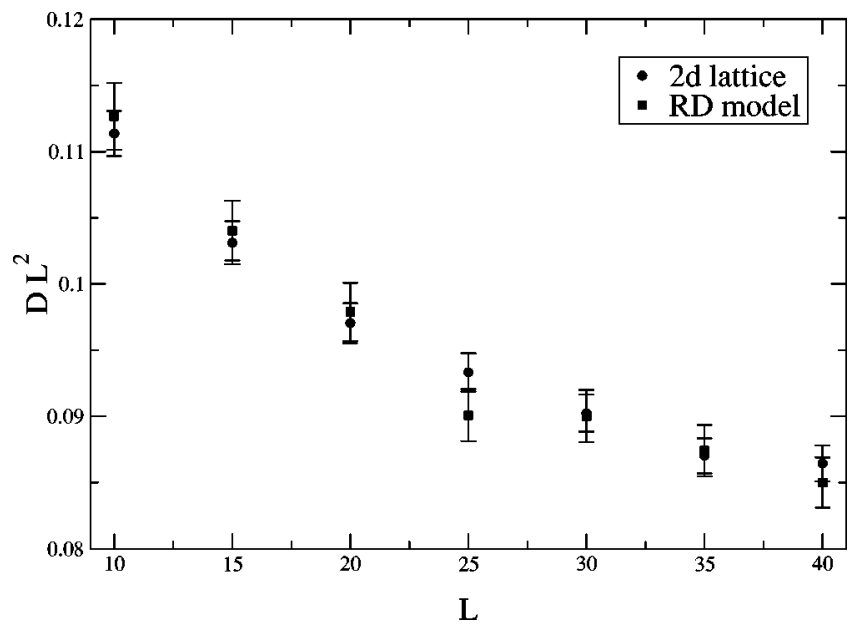

FIG. 2. Comparison between the diffusion coefficients for simulations of the disordered RD and two dimensional lattice model.

the actual local structure of the network. In order to verify that the model is correct on long time scales (as relevant for the center-of-mass diffusion), we performed Monte Carlo simulations comparing the diffusion constant of our projected model with $d=2$ at different disorder distributions with a polymer chain moving according to the dynamics of the repton model in a two-dimensional lattice with random but fixed hopping rates assigned to each cell boundary. It turns out that although the results differ for small chains ( $L$ $<10)$ they coincide within the statistical errors for longer chains. This legitimates our choice of boundary dynamics for investigating the behavior of long chains. Figure 2 shows an example where a binary disorder distribution with $W_{1}$ $=1 / 2, W_{2}=1 / 4$, and $\langle W\rangle=3 / 8$ was chosen.

Every move of a particle in the lattice gas leads to a change in the component of the center of mass coordinate of the repton chain along the field direction.

(1) Particle of type $\alpha$ moving to the right (left) decreases (increases) $x$ by $1 /(L+1)$, as this is equivalent to a repton moving downward (upward). As there are $L+1$ reptons, each contributes $1 /(L+1)$ to the center of mass position.

(2) Particle of type $-\alpha$ moving to the right (left) increases (decreases) $x$ by $1 /(L+1)$.

In the subsequent sections, we will calculate the drift velocity $v$ of the center of mass coordinate in presence of an applied electric field, and by employing the Einstein relation the zero-field diffusion constant. When calculating $v$, we restrict ourselves to the linear response regime, ignoring higher-order field dependences. In order to calculate $v$, only the change in the center of mass coordinate along the field $x$ has to be known, but not the absolute value itself. Whereas the latter cannot be known from the lattice gas, the former is given by the difference of the currents of positive particles $j^{+}$and that of the negative ones $j^{-}: v=j^{-}-j^{+}$. As the choice of the field direction is arbitrary, by use of the Einstein relation the model allows for computing the zero-field diffusion constant along a distinct direction in $d$-dimensional space. As zero-field diffusion is isotropic, this immediately yields the $d$-dimensional diffusion constant. This is due to distinguishing between particles of types $\alpha$ and $-\alpha$ in con- trast to the original projected repton model used by Rubinstein, which allows only for the computation of the curvilinear diffusion constant along the contour of the chain. In the Rubinstein model, additional assumptions are necessary to relate the curvilinear to the $d$-dimensional diffusion constant. Our model as well as the RD model allows for computation of the latter quantity within the model.

\section{QUANTUM HAMILTONIAN AND STATIONARY STATE}

The model introduced in the preceding section describes a Markov process and thus the dynamics can be written in form of a master equation. For convenience, we will use the quantum Hamiltonian formalism [20] to write down the master equation at zero electric field and solve for the stationary state.

At each site of the lattice gas with length $L, 2 \sigma+1$ values for $y_{i}$ are possible. Therefore, the state space $X$ has the dimension $(2 \sigma+1)^{L}$. Every $\eta \in X$ is assigned a vector $|\eta\rangle$ and a transposed vector $\langle\eta|$. These vectors constitute a basis for the space of system configurations $X$. A probability distribution $P(\eta) \equiv P_{\eta}$ can thus be written as a probability vector

$$
|P\rangle=\sum_{\eta \in X} P(\eta)|\eta\rangle
$$

Let a summation vector $\langle s|$ be defined as $\langle s|=\Sigma_{\eta \in X}\langle\eta|$. Normalization of the probability vector is given if $\langle s \mid P\rangle=1$. The generator for the dynamics of the system is the matrix $H$. The off-diagonal elements of the matrix contain the (negative) transition rates $t\left(\eta, \eta^{\prime}\right)$ from a state $\eta^{\prime}$ to $\eta$ :

$$
\left\langle\eta|H| \eta^{\prime}\right\rangle=H_{\eta, \eta^{\prime}}=-t\left(\eta, \eta^{\prime}\right) .
$$

The diagonal elements $H_{\eta, \eta}$ contain the sum of all the outgoing rates:

$$
\langle\eta|H| \eta\rangle=H_{\eta, \eta}=\sum_{\eta^{\prime} \neq \eta} t\left(\eta^{\prime}, \eta\right) .
$$

It is easily checked that conservation of probability, i.e., $\langle s| H=0$ in the language of the quantum Hamiltonian formalism, is fulfilled by $H$. The master equation

$$
\frac{d}{d t} P_{\eta}(t)=\sum_{\eta \neq \eta^{\prime}, \eta \in X}\left[t\left(\eta, \eta^{\prime}\right) P_{\eta^{\prime}}(t)-t\left(\eta^{\prime}, \eta\right) P_{\eta}(t)\right]
$$

describing the Markovian dynamics of the system can be written as

$$
\frac{d}{d t}|P(t)\rangle=-H|P(t)\rangle
$$

The stationary state $\left|P^{*}\right\rangle$ is thus characterized by the equation

$$
H\left|P^{*}\right\rangle=0
$$


In this formalism, the expectation value $\langle F(t)\rangle$ $=\Sigma_{\eta} F(\eta) P(\eta, t)$ of an operator $F$ is written as follows [20]. The operator $F: X \rightarrow X$ is represented by a diagonal matrix $F=\Sigma_{\eta} F(\eta)|\eta\rangle\langle\eta|$. Then,

$$
\langle F(t)\rangle=\left\langle s\left|F e^{-H t}\right| P(0)\right\rangle=\left\langle s\left|e^{H t} F e^{-H t}\right| P(0)\right\rangle .
$$

Let the time-dependent operator $F(t)$ for $t>0$ be defined as

$$
F(t)=e^{H t} F e^{-H t}
$$

$\langle F(t)\rangle$ is an expectation value that it is not only averaged over possible realizations of the process but also over the initial states according to $P(0)$. In the following, it is assumed that $|P(0)\rangle=\left|P^{*}\right\rangle$, so that

$$
\langle F(t)\rangle=\left\langle s|F(t)| P^{*}\right\rangle .
$$

For our model, we choose a tensor product basis as follows. Let at each site of the lattice gas the unit vector $e_{1}$ denote $y_{i}=0, e_{2 \alpha}$, denote $y_{i}=\alpha$ and $e_{2 \alpha+1}$ denote $y_{i}=-\alpha$. A state vector for a state $\eta=(1,-3, \ldots, 5,-2)$, for an example, then can be written as $|\eta\rangle=e_{2} \otimes e_{7} \otimes \cdots$ $\left.\otimes e_{10} \otimes e_{5}\right)$. Using this basis, the following operator creates a particle of type $\alpha$ at site $i$, provided it was previously unoccupied:

$$
a_{\alpha, 1}^{\dagger}(i)=\underbrace{\mathbf{1} \otimes \cdots \otimes}_{i-1} E_{(2 \alpha, 1)} \underbrace{\cdots \otimes \mathbf{1}}_{L-i}=E_{(2 \alpha, 1)}(i),
$$

where $E_{(2 \alpha, 1)}$ is the matrix with a single entry 1 at row $2 \alpha$ and column 1. Similarly, the operator $a_{\alpha,-1}^{\dagger}(i)$ $=E_{(2 \alpha+1,1)}(i)$ creates a particle of type $-\alpha$, if possible. The corresponding annihilation operators at site $i$ are $a_{\alpha, 1}(i)$ $=E_{(1,2 \alpha)}(i)$ and $a_{\alpha,-1}(i)=E_{(1,2 \alpha+1)}(i)$. To formulate the diagonal part of the quantum Hamiltonian, the matrices $u(i)$ $=E_{(1,1)}(i), \quad v_{\alpha, 1}(i)=E_{(2 \alpha, 2 \alpha)}(i) \quad$ and $\quad v_{\alpha,-1}(i)$ $=E_{(2 \alpha+1,2 \alpha+1)}(i)$ are employed. Thus, the quantum Hamiltonian for the model defined in the preceding section at zero field reads

$$
\begin{aligned}
H_{\text {open }}= & \sum_{i=1}^{L-1} \sum_{s= \pm 1} \sum_{\alpha=1}^{\sigma} W_{\alpha}\left[-a_{\alpha, s}^{\dagger}(i+1) a_{\alpha, s}(i)\right. \\
& -a_{\alpha, s}^{\dagger}(i) a_{\alpha, s}(i+1)+u(i+1) v_{\alpha, s}(i) \\
& \left.+u(i) v_{\alpha, s}(i+1)\right]+\sum_{s= \pm 1} \sum_{\alpha=1}^{\sigma}\left\{W_{\alpha} d f\left(W_{\alpha}\right)\right. \\
& \left.\times\left[-a_{\alpha, s}^{\dagger}(1)+u(1)\right]+W_{\alpha}\left[-a_{\alpha, s}(1)+v_{\alpha, s}(1)\right]\right\} \\
& +\sum_{s= \pm 1} \sum_{\alpha=1}^{\sigma}\left\{W_{\alpha} d f\left(W_{\alpha}\right)\left[-a_{\alpha, s}^{\dagger}(L)+u(L)\right]\right. \\
& \left.+W_{\alpha}\left(-a_{\alpha, s}(L)+v_{\alpha, s}(L)\right)\right\} .
\end{aligned}
$$

Plugging a product measure ansatz

$$
\begin{aligned}
\left|P_{\text {open }}^{*}(0)\right\rangle= & \left(\begin{array}{c}
1 \\
p_{1,1}(1) \\
p_{1,-1}(1) \\
p_{2,1}(1) \\
\vdots
\end{array}\right) \otimes \cdots \otimes\left(\begin{array}{c}
1 \\
p_{1,1}(L) \\
p_{1,-1}(L) \\
p_{2,1}(L) \\
\vdots
\end{array}\right) \\
& \times \frac{1}{\prod_{i=1}^{L}\left\{1+\sum_{\alpha=1}^{\sigma}\left[p_{\alpha, 1}(i)+p_{\alpha,-1}(i)\right]\right\}}
\end{aligned}
$$

into $H_{\text {open }}\left|P_{\text {open }}^{*}(0)\right\rangle=0$ leads to a very simple set of equations for the probabilities $p_{\alpha, \pm 1}$ and one finds

$$
\left|P_{\text {open }}^{*}(0)\right\rangle=\left(\begin{array}{c}
1 \\
d f\left(W_{1}\right) \\
d f\left(W_{1}\right) \\
\vdots \\
d f\left(W_{\sigma}\right) \\
d f\left(W_{\sigma}\right)
\end{array}\right)^{\otimes L} \frac{1}{(2 d+1)^{L}}
$$

The geometrical equilibrium conformation of the chain depends on the probability of occurrence for links between reptons along or against the field, irrespective of the assigned hopping rates of possibly crossed cell boundaries. This means we have to consider the overall probability for particles of positive sign at a site, which is $\sum_{\alpha=1}^{\sigma} f\left(W_{\alpha}\right) d /(2 d$ $+1)=d /(2 d+1)$, or negative sign, being also $d /(2 d+1)$, respectively. These are equal to the probabilities found for the disorder free RD model [21], which shows that the chosen kind of disorder is indeed kinematic disorder, as it influences only the mobility but not the equilibrium configurations of the chain.

Note that it is not possible to compute the stationary state for the model with a nonzero field at an arbitrary $L$. Therefore, the Einstein relation $(1 / L)(d / d E)(\langle v(E)\rangle)=D$ is not a feasible way of straightforwardly computing the diffusion constant. Extra input is needed to overcome this problem.

\section{MODEL WITH PERIODIC BOUNDARY CONDITIONS}

In this section, we adapt a calculation due to Kooiman and van Leeuwen [14] for the original RD model with periodic boundary conditions to the case with kinematic disorder. The quantum Hamiltonian for the periodic system at zero field reads

$$
\begin{aligned}
H_{p e r}= & \sum_{s= \pm 1} \sum_{\alpha=1}^{\sigma} \sum_{i=1}^{L} W_{\alpha}\left[-a_{\alpha, s}^{\dagger}(i+1) a_{\alpha, s}(i)\right. \\
& -a_{\alpha, s}^{\dagger}(i) a_{\alpha, s}(i+1)+u(i+1) v_{\alpha, s}(i) \\
& \left.+u(i) v_{\alpha, s}(i+1)\right] .
\end{aligned}
$$


Plugging $\left|P_{\text {open }}^{*}(0)\right\rangle$ as for the open system into the equation $H_{\text {per }}\left|P_{\text {open }}^{*}(0)\right\rangle=0$ for the periodic system shows that at zero electric field, the stationary state of the open system is also stationary with respect to the dynamics of a corresponding periodic system. In a periodic system, the phase space is nonergodic, as neither the order nor the number of occurring particles on such a ring can be changed. Therefore, every connected subset of the phase space ("channel") has its own stationary state. We can calculate the stationary state for the periodic system in presence of a field $E$ by mapping the system to a disordered zero-range process, as introduced by Benjamini, Ferrari, and Landim [22]. This means that the Einstein relation can be employed to obtain the diffusion constant in the periodic case. Here, the definition of the drift velocity of the center of mass and the corresponding diffusion constant are induced from the system with open boundary conditions: $v=j^{-}-j^{+}$.

Instead of characterizing the system by the spins on the lattice gas $\mathbf{y}=\left(y_{1}, \ldots, y_{L}\right)$, it can equivalently be characterized by the sets $\mathbf{S}=\left(s_{1}, \ldots, s_{L}\right)$ containing the signs of the nonzero $y_{i}, \mathbf{w}=\left(w_{1}, \ldots, w_{L}\right)$ containing the absolute values of these $y_{i}$ and $\mathbf{n}=\left(n_{1}, \ldots, n_{L}\right)$ where $n_{i}$ amounts to the number of " 0 " between $s_{i}$ and $s_{i+1}$ on the lattice gas. Thus, every lattice gas configuration on a ring can be translated into a configuration on a lattice of length $M$ in the zero-range picture. Site $i$ carries $n_{i}$ zero range particles and is separated from site $i+1$ by a bond characterized by $s_{i+1}$ and $w_{i+1}$. The total number $K$ of zero-range particles must equal the total number $L-M$ of 0 in the lattice gas: $\sum_{i=1}^{M} n_{i}=K=L$ $-M$. The dynamics of the lattice gas picture translates into the zero range picture as follows. The configuration $\left(\ldots, n_{j}, n_{j+1}, \ldots\right)$ changes to $\left(\ldots, n_{j}-1, n_{j+1}+1, \ldots\right)$ with rate $h_{j+1}^{-1} w_{j+1}$ and to $\left(\ldots, n_{j}+1, n_{j+1}-1, \ldots\right)$ with rate $h_{j+1} w_{j+1}$, where $h_{j}=\exp \left(-E s_{j} / 2\right)$. This means that the random hopping rates, as well as the $s_{j}$, are not assigned to individual particles, but to bonds between sites in the zerorange (zr) picture. At $E=0$, zr particles move as in a random barrier energy landscape. Moves of the zr particles cause changes of the center of mass coordinate as defined above. A zr-particle hopping to the right across a bond with $s_{j}>0$ $\left(s_{j}<0\right)$ increases (decreases) the center of mass position by $1 / L$. Conversely, a zr-particle hopping to the left across a bond with $s_{j}>0\left(s_{j}<0\right)$ decreases (increases) the center of mass position by $1 / L$. As with the field free case of the open system, we use a quantum Hamiltonian and a tensor basis for the state space to compute the stationary state of the zr lattice of length $M$ with a given $\mathbf{s}$ and $\mathbf{w}$. Let $e_{1}(i)$ denote an unoccupied site $i$ and $e_{m}(i)$ where $m>1$ a site $i$ occupied by $n_{i}=m-1$ particles. Here, vectors are infinite dimensional. The matrix for the creation of a particle at site $i$ is then given by

$$
b_{i}^{\dagger}=\left(\begin{array}{ccccc}
0 & 0 & \ldots & 0 & \ldots \\
1 & 0 & \ldots & 0 & \ldots \\
\vdots & \ddots & \ddots & \vdots & \ldots \\
0 & & 1 & 0 & \ldots \\
\vdots & \ldots & \ldots & \ddots & \ddots
\end{array}\right)_{i}
$$

The matrix for the annihilation of a particle at site $i$ is accordingly given by

$$
b_{i}=\left(\begin{array}{ccccc}
0 & 1 & \ldots & 0 & \ldots \\
0 & 0 & 1 & \ldots & \ldots \\
\vdots & \ddots & \ddots & \ddots & \ldots \\
0 & & 0 & 0 & \ddots \\
\vdots & \ldots & \ldots & \ldots & \ddots
\end{array}\right)_{i}
$$

For constructing the diagonal part of the Hamiltonian, we need the following type of matrix (note that $\langle s| b_{i}^{\dagger}=\langle s| \mathbf{1}$ and $\left.\langle s| b_{i}=\langle s| m_{i}\right)$ :

$$
m_{i}=\left(\begin{array}{ccccc}
0 & 0 & \ldots & \ldots & \ldots \\
0 & 1 & \ddots & & \ldots \\
\vdots & \ddots & \ddots & 0 & \ldots \\
\vdots & & 0 & 1 & \ddots \\
\vdots & \ldots & \ldots & \ddots & \ddots
\end{array}\right)_{i}
$$

A hopping event from site $i$ to site $i+1$ is described by the combined action of the matrices $b_{i}$ and $b_{i+1}^{\dagger}$ This yields the following expression for the quantum Hamiltonian:

$$
\begin{aligned}
H_{z r}= & \sum_{i=1}^{M}\left(-h_{i+1} b_{i+1} b_{i}^{\dagger} w_{i+1}-h_{i+1}^{-1} b_{i} b_{i+1}^{\dagger} w_{i+1}\right. \\
& \left.+h_{i+1}^{-1} w_{i+1} m_{i}+h_{i+1} w_{i+1} m_{i+1}\right) .
\end{aligned}
$$

A yet unnormalized product ansatz

$$
\left|P_{z r}^{*}\right\rangle=\left(\begin{array}{c}
1 \\
z_{1} \\
z_{1}^{2} \\
\vdots
\end{array}\right) \otimes \cdots \otimes\left(\begin{array}{c}
1 \\
z_{M} \\
z_{M}^{2} \\
\vdots
\end{array}\right)
$$

yields

$$
\begin{aligned}
H_{z r}\left|P_{z r}^{*}\right\rangle= & \sum_{i=1}^{M}\left(-h_{i+1} w_{i+1} \frac{z_{i+1}}{z_{i}} m_{i}\left|P_{z r}^{*}\right\rangle\right. \\
& -h_{i+1}^{-1} w_{i+1} \frac{z_{i}}{z_{i+1}} m_{i+1}\left|P_{z r}^{*}\right\rangle+h_{i+1}^{-1} w_{i+1} m_{i}\left|P_{z r}^{*}\right\rangle \\
& \left.+h_{i+1} w_{i+1} m_{i+1}\left|P_{z r}^{*}\right\rangle\right) \\
= & \sum_{i=1}^{M}\left(-h_{i+1} w_{i+1} \frac{z_{i+1}}{z_{i}} m_{i}\left|P_{z r}^{*}\right\rangle\right. \\
& -h_{i}^{-1} w_{i} \frac{z_{i-1}}{z_{i}} m_{i}\left|P_{z r}^{*}\right\rangle+h_{i+1}^{-1} w_{i+1} m_{i}\left|P_{z r}^{*}\right\rangle \\
& \left.+h_{i} w_{i} m_{i}\left|P_{z r}^{*}\right\rangle\right) .
\end{aligned}
$$


$\left|P_{z r}^{*}\right\rangle$ is stationary provided

$$
h_{i+1} w_{i+1} z_{i+1}+h_{i}^{-1} w_{i} z_{i-1}=h_{i+1}^{-1} w_{i+1} z_{i}+h_{i} w_{i} z_{i} .
$$

Applying a general solution [23] to the case at hand and including normalization, the stationary state for a channel characterized by $\mathbf{s}$ and $\mathbf{w}$ and with a total number of particles $K$ yields

$$
\begin{aligned}
\left|P_{z r}^{*}(\mathbf{s}, \mathbf{w}, K, M)\right\rangle= & \sum_{\left(n_{1}, \ldots, n_{M}\right)}^{\prime} \prod_{i=1}^{M} z_{i}^{n_{i}}\left|n_{1}, \ldots, n_{M}\right\rangle \\
& \times \frac{1}{\sum_{\left(n_{1}, \ldots, n_{M}\right)}^{\prime} \prod_{i=1}^{M} z_{i}^{n_{i}}}
\end{aligned}
$$

where

$$
z_{l}=\sum_{i=1}^{M} \frac{1}{h_{l+i}^{-1} w_{l+i}} \prod_{j=1}^{i-1} h_{l+j}^{2} .
$$

The primed sums are meant to be summations under the constraint $\sum_{i=1}^{\sigma} n_{i}=K$. Knowing the stationary state for each channel, the drift velocity for the individual channels can be computed. In the lattice gas picture, every time a particle $s_{i}$ $=-1\left(s_{i}=1\right)$ is hopping to the right (left), it changes the position of the center of mass by $1 /(L+1)$. The opposite process, i.e., a particle $s_{i}=-1 \quad\left(s_{i}=1\right)$ hopping to the left (right) changes the center of mass position by $-1 /(L+1)$. Therefore, as mentioned above, $v=j^{-}-j^{+}$, i.e., the difference between the currents of negative and positive particles. This operator $v$ translates to the $\mathrm{zr}$ picture as follows:

$$
j^{-}-j^{+} \rightarrow \frac{1}{L} \sum_{i=1}^{M} s_{i+1} w_{i+1}\left(h_{i+1}^{-1} b_{i} b_{i+1}^{\dagger}-h_{i+1} b_{i+1} b_{i}^{\dagger}\right) .
$$

Thus, the drift velocity of a given channel is given by

$$
\begin{aligned}
&\langle v(\mathbf{s}, \mathbf{w}, K, M)\rangle=\langle s| \frac{1}{L} \sum_{i=1}^{M}\left(s_{i+1} h_{i+1}^{-1} w_{i+1} b_{i} b_{i+1}^{\dagger}\right. \\
&\left.-s_{i+1} h_{i+1} w_{i+1} b_{i+1} b_{i}^{\dagger}\right) \Theta(K)\left|P_{z r}^{*}\right\rangle \frac{1}{c_{K}} .
\end{aligned}
$$

Here, $\Theta(K)$ projects on those states of $\left|P_{z r}^{*}\right\rangle$ which have a constant number of particles $K$ and $c_{K}$ is the normalization. So, $\Theta(K) / c_{K}\left|P_{z r}^{*}\right\rangle=\left|P_{z r}^{*}(\mathbf{s}, \mathbf{w}, K)\right\rangle$. Using this form allows us to make use of the fact that due to the combined effect of $b_{i} b_{j}^{\dagger}$, which is to redistribute the particles without changing their number, we can commute $b_{i} b_{j}^{\dagger}$ and $\Theta(K)$. This allows us then to apply the matrices to the product measure, where its effect is easy to see,

$$
\begin{aligned}
&\langle v(\mathbf{s}, \mathbf{w}, K, M)\rangle=\langle s| \frac{1}{L} \sum_{i=1}^{M}\left(s_{i+1} h_{i+1}^{-1} w_{i+1} b_{i} b_{i+1}^{\dagger}\right. \\
&\left.-s_{i+1} h_{i+1} w_{i+1} b_{i+1} b_{i}^{\dagger}\right) \Theta(K)\left|P_{z r}^{*}\right\rangle \frac{1}{c_{K}} \\
&=\langle s| \frac{1}{L} \sum_{i=1}^{M}\left[s_{i+1} h_{i+1}^{-1} w_{i+1} \Theta(K) b_{i} b_{i+1}^{\dagger}\right. \\
&\left.-s_{i+1} h_{i+1} w_{i+1} \Theta(K) b_{i+1} b_{i}^{\dagger}\right]\left|P_{z r}^{*}\right\rangle \frac{1}{c_{K}} \\
&= \frac{1}{L} \sum_{i}\left[s_{i+1} h_{i+1}^{-1} w_{i+1} \frac{z_{i}}{z_{i+1}}\right. \\
& \times\left\langle s\left|m_{i+1}\right| \Theta(K) P_{z r}^{*}\right\rangle-s_{i+1} h_{i+1} w_{i+1} \\
&\left.\times \frac{z_{i+1}}{z_{i}}\left\langle s\left|m_{i}\right| \Theta(K) P_{z r}^{*}\right\rangle\right] \frac{1}{c_{K}} .
\end{aligned}
$$

Now, we have to calculate the expression $c_{K}$ for the product state:

$$
c_{K}=\sum_{\left(n_{1}, \ldots, n_{M}\right)}^{\prime} \prod_{i=1}^{M} z_{i}^{n_{i}},
$$

where the primed sum again means summing with the constraint $\sum_{i=1}^{M} n_{i}=K$.

A similar type of summation is found when regarding the sums of the type

$$
\left\langle s\left|m_{\beta} \frac{\Theta(K)}{c_{K}}\right| P_{z r}^{*}\right\rangle=\sum_{\left(n_{1}, \ldots, n_{M}\right)}^{\prime \prime} \frac{1}{c_{K}} \prod_{j=1}^{M} z_{j}^{n_{j}}
$$

where the double primed sum has the constraints $\sum_{j=1}^{M} n_{j}$ $=K$ and $n_{\beta} \neq 0$. A straightforward calculation of the sums is impossible due to the constraints. To simplify the task, we can profitably use the following identity [14]:

$$
\sum_{\left(n_{1}, \ldots, n_{M}\right)}^{\prime} \prod_{i=1}^{M} z_{i}^{n_{i}}=\frac{1}{2 \pi i} \oint \frac{d \alpha}{\alpha^{K+1}} \sum_{\left(n_{1}, \ldots, n_{M}\right)} \prod_{j=1}^{M}\left(\alpha z_{j}\right)^{n_{j}} .
$$

Thus, we can transform the sums into integrals:

$$
\begin{aligned}
c_{K} & =\frac{1}{2 \pi i} \oint \frac{d \alpha}{\alpha^{K+1}} \sum_{\left(n_{1}, \ldots, n_{M}\right)} \prod_{j=1}^{M}\left(\alpha z_{j}\right)^{n_{j}} \\
& =\frac{1}{2 \pi i} \oint \frac{d \alpha}{\alpha^{K+1}} \prod_{j=1}^{M} \frac{1}{1-\alpha z_{j}} \\
& =Q_{K, M},
\end{aligned}
$$




$$
\begin{aligned}
& \left\langle s\left|m_{\beta} \frac{\Theta(K)}{c_{K}}\right| P_{z r}^{*}\right\rangle \\
& =\frac{1}{c_{K}} \frac{1}{2 \pi i} \oint \frac{d \alpha}{\alpha^{K+1}} \sum_{\left(n_{1}, \ldots, n_{M}\right), n_{\beta} \neq 0} \prod_{j=1}^{M}\left(\alpha z_{j}\right)^{n_{j}} \\
& =\frac{1}{c_{K}} z_{\beta} \frac{1}{2 \pi i} \oint \frac{d \alpha}{\alpha^{K}} \prod_{j=1}^{M} \frac{1}{1-\alpha z_{j}} \\
& =z_{\beta} \frac{Q_{K-1, M}}{Q_{K, M}} .
\end{aligned}
$$

The integrals $Q_{K, M}$ satisfy the recursion relation,

$$
Q_{K, M}=Q_{K, M-1}+z_{M} Q_{K-1, M}
$$

An explicit solution of this relation yields

$$
Q_{K, M}=\sum_{i=1}^{M} z_{i}^{K+M-1} \prod_{l=1}^{M^{\prime}}\left(z_{i}-z_{l}\right)^{-1}
$$

The primed product indicates $i \neq l$. From this point onwards, we are not going to carry through the complete calculation for $\langle v(\mathbf{s}, \mathbf{w}, K, M)\rangle$. We will expand it into a series in $E$ and keep only first-order terms, which is sufficient for employing the Einstein relation. We start from the expression for $\langle v(\mathbf{s}, \mathbf{w}, K, M)\rangle$ after having inserted the result for $\left\langle s\left|m_{i} \Theta(N-L) / c_{K}\right| P_{z r}^{*}\right\rangle$ :

$$
\begin{aligned}
\langle v(s, w, K)\rangle= & \frac{1}{L} \sum_{i=1}^{M} s_{i+1} h_{i+1}^{-1} w_{i+1} \frac{z_{i}}{z_{i+1}} z_{i+1} \frac{Q_{K-1, M}}{Q_{K, M}} \\
& -s_{i+1} h_{i+1} w_{i+1} \frac{z_{i+1}}{z_{i}} z_{i} \frac{Q_{K-1, M}}{Q_{K, M}} \\
= & \frac{1}{L} \sum_{i=1}^{M} s_{i+1}\left(h_{i+1}^{-1} w_{i+1} z_{i}\right. \\
& \left.-h_{i+1} w_{i+1} z_{i+1}\right) \frac{Q_{K-1, M}}{Q_{K, M}} \\
= & \frac{1}{L} \sum_{i=1}^{M} s_{i+1}[1-\exp (-E S)] \frac{Q_{K-1, M}}{Q_{K, M}} \\
= & \frac{S}{L}[1-\exp (-E S)] \frac{Q_{K-1, M}}{Q_{K, M}},
\end{aligned}
$$

where $S=\sum_{i=1}^{M} s_{i}$. For the second equality, we used the explicit form of the $z_{i}$. The term in brackets is easily expanded into a series in $E: 1-\exp (E S)=E S+O\left(E^{2}\right)$. We keep only the first-order term in $E$. This means that when we are expanding the expression $Q_{K-1, M} / Q_{K, M}$ we need only to keep zeroorder terms in $E$, as all other contributions will vanish when using the Einstein relation. The only terms containing $E$ in the $Q_{K, M}$ are the $z_{i}$, for which we find to first order in $E$ :

$$
\begin{aligned}
z_{i} & =\sum_{j=1}^{M} \frac{1}{h_{i+j}^{-1} w_{i+j}} \prod_{k=1}^{j-1} h_{i+k}^{2} \\
& =\sum_{j=1}^{M} \frac{1}{w_{i+j}}\left(1-\frac{E}{2} s_{j+i}+\cdots\right) \prod_{k=1}^{j-1}\left(1-E s_{i+k}+\cdots\right) \\
& =\sum_{j=1}^{M} \frac{1}{w_{i+j}}+O(E)=z+O(E)
\end{aligned}
$$

Here, $z$ is defined as the zero field value of $z_{i}$. The fact that all $z_{i}$ are equal at zero field is decisive for the explicit integration of $Q_{K, M}$. We obtain

$$
Q_{K, M}=\frac{1}{2 \pi i} \oint \frac{d \alpha}{\alpha^{K+1}} \prod_{j=1}^{M} \frac{1}{1-\alpha z_{j}}=\left(\begin{array}{c}
M+K-1 \\
M-1
\end{array}\right) z^{K} .
$$

It is now clear how to proceed with evaluating $\langle v(s, w, K, M)\rangle$, as

$$
\frac{Q_{K-1, M}}{Q_{K, M}}=\frac{\left(\begin{array}{c}
K+M-2 \\
M-1
\end{array}\right) z^{M-1}}{\left(\begin{array}{c}
K+M-1 \\
M-1
\end{array}\right) z^{K}}=\frac{K}{K+M-1} \frac{1}{z} .
$$

This yields for $\langle v(s, w, K, M)\rangle$ :

$$
\begin{aligned}
\langle v(\mathbf{s}, \mathbf{w}, K, M)\rangle & =\frac{1}{L} \sum_{i=1}^{M} s_{i+1} E S \frac{K}{K+M-1} \frac{1}{z}+O\left(E^{2}\right) \\
& =\frac{1}{L} E S^{2} \frac{K}{K+M-1} \frac{1}{z}+O\left(E^{2}\right) .
\end{aligned}
$$

This is our final expression for $\langle v(\mathbf{s}, \mathbf{w}, K, M)\rangle$. Knowing the drift velocity for each channel characterized by $\mathbf{s}, \mathbf{w}$, we have to give each of these a weight $\Psi(\mathbf{s}, \mathbf{w}, K, M)$ when averaging over the channels. Following an argument by Prähofer [21], we show below that provided a configuration in the $\mathrm{zr}$ picture is weighted such that at $E=0$, its probability as contained in $P_{\text {ring }}^{*}(\mathbf{s}, \mathbf{w}, K, M)=\Psi(\mathbf{s}, \mathbf{w}, K, M) P_{z r}^{*}(\mathbf{s}, \mathbf{w}, K, M)$ is equal to the probability of the corresponding state in the lattice gas of the open system as given by $P_{\text {open }}^{*}(0)$, for the diffusion constants the following relation holds: $D_{L+1}^{\text {open }}$ $\geqslant D_{L}^{\text {per }}$.

When relating zr to lattice gas probabilities, it has to be kept in mind that the periodic $\mathrm{zr}$ system has $M$ sites, while the corresponding periodic lattice gas has $L \geqslant M$ sites. This means that a state of the zr system with probability $q_{z r}$ can be permutated $M$ times, where due to translational invariance all resulting states have the same probability. A lattice gas configuration with probability $q_{l g}$ can be cycled through $L$ permutations leading to equal probabilities. Therefore, equal probabilities of respective configurations means $M q_{z r}$ $=L q_{l g}$. The weight factor thus has to be chosen as follows: 


$$
\Psi(\mathbf{s}, \mathbf{w}, K, M)=\frac{d^{M}}{(2 d+1)^{L}}\left(\begin{array}{c}
L \\
M
\end{array}\right) \prod_{j=1}^{M} f\left(w_{j}\right)
$$

Thus, we find for the drift velocity, when averaged over the channels,

$$
\begin{aligned}
\bar{v} & =\sum_{M} \sum_{\mathbf{s}=\left(s_{1}, \ldots, s_{M}\right) \quad \mathbf{w}^{\prime}=\left(w_{1}, \ldots, w_{M}\right)} \Psi(\mathbf{s}, \mathbf{w})\langle v(\mathbf{s}, \mathbf{w}, K, M)\rangle \\
& =\sum_{M} \frac{1}{L} E M \frac{d^{M}}{(2 d+1)^{L}}\left(\begin{array}{c}
L \\
M
\end{array}\right) \frac{L-M}{L-1}\left(\sum_{w} \prod_{j=1}^{M} f\left(w_{j}\right) \frac{1}{z}\right) .
\end{aligned}
$$

This can be rewritten as

$$
\bar{v}=E \sum_{M=1}^{L} \Omega(M) M\left\langle\frac{1}{z}\right\rangle
$$

Here, $\Omega(M)$ contains all the factors depending on $M$ occurring in the previous expression and $\langle 1 / z\rangle$ is a disorder average. $\Omega(M)$ is a function that is sharply peaked at $M$ $=L / 2$ implying that in the limit for large $L$, only terms with large $M$ significantly contribute to the result. In the limiting case of $L \rightarrow \infty$ invoking the central limit theorem (remember that we demanded $\langle 1 / W\rangle$ and $\left\langle 1 / W^{2}\right\rangle$ to be finite) yields

$$
\left\langle\frac{1}{z}\right\rangle=\frac{1}{M\langle 1 / W\rangle}
$$

Therefore,

$$
\bar{v}=\sum_{M=1}^{L} \frac{1}{L} E M \frac{d^{M}}{(2 d+1)^{L}}\left(\begin{array}{c}
L \\
M
\end{array}\right) \frac{L-M}{L-1} \frac{1}{M} \frac{1}{\left\langle\frac{1}{W}\right\rangle}
$$

Using the Einstein relation yields in the limit for large $L$

$$
\begin{aligned}
D(0) & =\frac{1}{L(L-1)} \frac{1}{(2 d+1)}\left(1-\frac{1}{(2 d+1)^{L-1}}\right) \frac{1}{\left\langle\frac{1}{W}\right\rangle} \\
& =D^{*} \frac{1}{\left\langle\frac{1}{W}\right\rangle}
\end{aligned}
$$

where $D^{*}$ is the diffusion constant for the ordered case $[14,15]$.

Thus, we have shown that $\lim _{L \rightarrow \infty} D L^{2}=1 /$ $[(2 d+1)\langle 1 / W\rangle]$ for the periodic case, which is a lower bound to the open case. Note that the factor $1 /\langle 1 / W\rangle$ is the same as occurring in the single particle diffusion constant for the random barrier model. In this model, random energy barriers are assigned to bonds between sites just as in the $\mathrm{zr}$ picture of our problem.

\section{MODEL WITH OPEN BOUNDARY CONDITIONS}

\section{A. Variational formula}

In order to find an upper bound on the diffusion constant, we follow the strategy of Ref. [15]. The quantum Hamiltonian $H_{\text {open }}$ for our model in its representation using the tensor basis can be decomposed into the sum of a diagonal part $D$ and a nondiagonal one $M$. Each of these can themselves be split into a part describing those moves leading to an increase of the center of mass coordinate $\left(M^{+}\right.$and $\left.D^{+}\right)$ and a respective part connected to a decrease $\left(M^{-}\right.$and $\left.D^{-}\right)$. Thus, $H=D^{+}+D^{-}-M^{+}-M^{-}$.

The following statement [24] holds for the diffusion constant $D$ :

$$
\begin{aligned}
D= & \inf _{g \in \Omega}\left[\frac{1}{2(L+1)^{2}}\left\langle D^{+}+D^{-}\right\rangle\right. \\
& \left.-\frac{2}{(L+1)}\left\langle s\left|\left[D^{+}-D^{-}\right] g\right| P^{*}\right\rangle+\left\langle s|g H g| P^{*}\right\rangle\right] \\
= & \inf _{g \in \Omega} F[g] .
\end{aligned}
$$

Here, $\Omega$ is the space of diagonal matrices with dimension $(2 \sigma+1)^{L}$. Thus, plugging in any diagonal matrix $g$ into the functional $F[g]$ yields an upper bound on $D$. The challenge is to choose $g$ such that the upper bound gets as small as possible. Due to the dimensionality of the diagonal matrices increasing exponentially with $L$, an exact minimization is not feasible. Still, some information can be gained by observing that the functional is convex and thus the minimizing $g_{0}$ $\in \Omega$ is the only matrix for which $\delta F[g]$ vanishes. Choosing $g_{0}$ such that

$$
\langle s| g_{0} H=\langle s|\left[D^{+}-D^{-}\right],
$$

the variation vanishes. Unfortunately, this formula cannot be solved for $g_{0}$. We will show below how still some insight can be gained from this equation. Introducing the matrix $d$, where $d\left(y^{\prime} \mid y\right)=\left\langle y^{\prime}|d| y\right\rangle$ gives the change in the CMS coordinate when a transition from state $y$ to $y^{\prime}$ is made, and the matrix $w$, where $w\left(y^{\prime} \mid y\right)$ denotes the corresponding rate, the variational formula may be written as:

$$
D=\inf \frac{1}{2} \sum_{y^{\prime}, y \in X} w\left(y^{\prime} \mid y\right) P^{*}(y)\left[d\left(y^{\prime} \mid y\right)+g\left(y^{\prime}\right)-g(y)\right]^{2},
$$

where $g(y)=\langle s|g| y\rangle$. Following Ref. [21], we now prove that $D_{L}^{\text {open }} \geqslant D_{L+1}^{\text {per }}$. To simplify the notation, we remark that in our model with periodic boundary conditions as in the original RD model, transitions from a given state $y$ are only allowed to a state $y^{\prime}=y^{i, i+1}$, where the spins $y_{i}$ and $y_{i+1}$ are interchanged. Thus, the last formula applied to the periodic case reads as 


$$
\begin{aligned}
D_{L+1}^{p e r}= & \inf \frac{1}{2} \sum_{l=0}^{L}\left(\sum_{y \in X} w\left(y^{l, l+1} \mid y\right) P^{*}(y)\right. \\
& \left.\times\left[d\left(y^{l, l+1} \mid y\right)+g\left(y^{l, l+1}\right)-g(y)\right]^{2}\right) .
\end{aligned}
$$

Here, each transition between states in a lattice gas of length $L+1$ changes the $\mathrm{cm}$ coordinate by $\pm 1 /(L+1)$, as there are as many reptons as bonds between them. In contrast to this, in the open boundary case, there is one more repton as there are bonds leading to the $\mathrm{cm}$ coordinate changing by $\pm 1 /(L+1)$ in any transition of a lattice gas of length $L$. Here, transitions between states can not only occur by interchanging spins $y_{1}$ and $y_{i+1}$ in the bulk but also by creation and annihilation events at the ends. These events can equivalently be viewed as interchanges of the spins $y_{1}$ and $y_{L}$, respectively, with "imaginary" spins $y_{0}$ and $y_{L+1}$ provided the rates for these interchanges at the ends are adapted such that these correspond to the creation and annihilation rates as demanded by the model. Taking the distribution at the "imaginary" sites as for any other site in the lattice gas (remember that we have a homogeneous product measure), the introduction of a parameter

$$
c_{l}=\left\{\begin{array}{lll}
2 d+1 & \text { if } & l=0 \text { or } l=L \\
1 & \text { for } & l=1, \ldots, L-1
\end{array}\right.
$$

leads to a fulfillment of this requirement, and the functional for the diffusion constant of the open chain of length $L$ reads as

$$
\begin{aligned}
D_{L}^{\text {open }}= & \inf \frac{1}{2} \sum_{l=0}^{L} c_{l}\left(\sum _ { y \in X } w ( y ^ { l , l + 1 } | y ) P ^ { * } ( y ) \left[d\left(y^{l, l+1} \mid y\right)\right.\right. \\
& \left.\left.+g\left(y^{l, l+1}\right)-g(y)\right]^{2}\right),
\end{aligned}
$$

where $w$ and $d$ are as for the periodic case. Letting $\mathbf{y}$ $=\left(y_{0}, \ldots, y_{L+1}\right)$ for open and periodic system, and observing that as $y_{0}$ does not occur in the functional for $D_{L+1}^{p e r}$, the averaging of $y_{0}$ over $P^{*}$ yields 1 and thus does not change the result:

$$
\begin{aligned}
& D_{L}^{\text {open }}[g]-D_{L+1}^{\text {per }}[g] \\
&=c_{0}\left(\sum_{y \in X} w\left(y^{0,1} \mid y\right) P^{*}(y)\left[d\left(y^{0,1} \mid y\right)+g\left(y^{0,1}\right)-g(y)\right]^{2}\right) \\
&+c_{L}\left(\sum _ { y \in X } w ( y ^ { L , L + 1 } | y ) P ^ { * } ( y ) \left[d\left(y^{L, L+1} \mid y\right)\right.\right. \\
&\left.\left.+g\left(y^{L, L+1}\right)-g(y)\right]^{2}\right)-\sum_{y \in X} w\left(y^{L+1,1} \mid y\right) P^{*}(y) \\
& \times\left[d\left(y^{0,1} \mid y\right)+g\left(y^{0,1}\right)-g(y)\right]^{2} \\
&-\sum_{y \in X} w\left(y^{L, L+1} \mid y\right) P^{*}(y)
\end{aligned}
$$

$$
\begin{aligned}
& \times\left[d\left(y^{L, L+1} \mid y\right)+g\left(y^{L, L+1}\right)-g(y)\right]^{2} \\
= & 2 \sum_{y \in X} w\left(y^{L+1,1} \mid y\right) P^{*}(y)\left[d\left(y^{0,1} \mid y\right)+g\left(y^{0,1}\right)-g(y)\right]^{2} \\
& +2 \sum_{y \in X} w\left(y^{L, L+1} \mid y\right) P^{*}(y)\left[d\left(y^{L, L+1} \mid y\right)\right. \\
& \left.+g\left(y^{L, L+1}\right)-g(y)\right]^{2} \\
\geqslant & 0 .
\end{aligned}
$$

Therefore, the diffusion constant of the periodic chain with length $L+1$ is a lower bound for the open chain with length $L$.

\section{B. Functional for diffusion with kinematic disorder}

The space of diagonal matrices $\Omega$, from which an appropriate $g \in \Omega$ has to be chosen such that $F[g]$ gets as small as possible, has dimension $(2 \sigma+1)^{L}$. A scalar product on $\Omega$ can be defined for arbitrary $m, n \in \Omega$ as $\left\langle s|m n| P^{*}\right\rangle$. A basis for $\Omega$ can be built from the matrices $\hat{y}_{1}(i)$ to $\hat{y}_{2 \sigma}(i)$ acting nontrivially only at site $i$ and the unit matrix. We specify only the following:

$$
\begin{aligned}
& \hat{y}_{\alpha}(i)=\frac{1}{2 d+1} E_{(2 \alpha, 2 \alpha)}(i)-\frac{1}{2 d+1} E_{(2 \alpha+1,2 \alpha+1)}(i) \\
& \text { for } \alpha=1, \ldots, \sigma, \\
& \hat{y}_{\sigma+1}(i)=-\frac{2 d}{2 d+1} E_{(1,1)}(i)+\sum_{j=2}^{2 \sigma+1} \frac{1}{2 d+1} E_{(j, j)}(i) .
\end{aligned}
$$

For the remaining $\sigma-1$ matrices, we demand that these are chosen such that for all $a, b=1, \ldots, 2 \sigma, i, j=1, \ldots, L$, and $a \neq b$, we have $\left\langle\hat{y}_{a}(i) \hat{y}_{b}(j)\right\rangle=0$ as well as $\left\langle\hat{y}_{a}(i)\right\rangle=0$. It is easily checked that the defined matrices are mutually orthogonal. A basis for the space of diagonal matrices $\Omega$ is then given by

$$
\left\{\prod_{j=1}^{2 \sigma+1} \hat{y}_{j}\left(I_{j}\right) \mid I_{j} \subset\{1, \ldots, L\} \quad \forall j ; I_{\alpha} \cap I_{\beta}=\oslash \quad \forall \quad \alpha \neq \beta\right\} .
$$

The choice of a $g \in \Omega$ fulfilling $\langle s|\left[D^{+}-D^{-}\right]=\langle s| g H$ leads to $\delta F[g]=0$. In spite of this relation not being solvable for $g$, information can be gained by observing that $\Omega$ contains subsets $\Omega_{i_{1}}, \ldots, i_{\sigma}$ which are invariant to $H$ in the sense that if $g \in \Omega_{i_{1}}, \ldots, i_{\sigma}$, then the diagonal matrix $g^{\prime}$ fulfilling $\langle s| g H=\langle s| g^{\prime}$ is in $\Omega_{i_{1}, \ldots, i_{\sigma}}$ too, where

$$
\begin{aligned}
\Omega_{i_{1}, \ldots, i_{\sigma}}= & \operatorname{span}\left\{\prod_{j=1}^{2 \sigma+1} \hat{y}_{j}\left(I_{j}\right) \mid I_{j} \subset\{1, \ldots,\}\right. \\
& \forall j ; I_{\alpha} \cap I_{\beta}=\oslash \quad \forall \alpha \neq \beta ;\left|I_{j}\right|=i_{j} \\
& \forall j=1, \ldots, \sigma\} .
\end{aligned}
$$


By calculating $\langle s|\left[D^{+}-D^{-}\right]$, we find that the minimizing $g_{0}$ is in the subspace $\oplus_{j=1}^{\sigma} \Omega_{i_{1}, \ldots, i_{\sigma}}$ where, $i_{k}$ $=\delta_{i, k} \forall k=1, \ldots, \sigma$. Thus, the most general ansatz for $g$ based on this information is

$$
\begin{aligned}
g= & \sum_{\alpha=1}^{\sigma} \sum_{i=0}^{L+1} \sum_{I_{\sigma+1}, \ldots, I_{2 \sigma+1}} c_{i, I_{\sigma+1}}^{\alpha} \ldots, I_{2 \sigma+1} \hat{y}_{\alpha}(i) \\
& \times \hat{y}_{\sigma+1}\left(I_{\sigma+1}\right) \cdots \hat{y}_{2 \sigma+1}\left(I_{2 \sigma+1}\right),
\end{aligned}
$$

where the coefficients $c$ are real numbers and the sets $I_{\sigma}, \ldots, I_{2 \sigma+1}$ are mutually disjoint.

\section{Ansatz for $g$}

The general ansatz given above for $g$ contains too many parameters for letting a minimization appear feasible. Instead, we use a generalization of the ansatz used by Prähofer [21] for the ordered RD model:

$$
\begin{aligned}
g= & \sum_{\alpha=1}^{\sigma}\left\{\sum_{i=0}^{L+1} a_{i}^{\alpha} \hat{y}_{\alpha}(i)+\sum_{k, k^{\prime}=0}^{L+1}{ }^{\prime} c_{k, k^{\prime}}^{\alpha} \hat{y}_{\alpha}(k) \hat{y}_{\sigma+1}\left(k^{\prime}\right)\right. \\
& \left.+\sum_{o, p, q=0 ; p<q}^{L+1} e_{o, p, q}^{\alpha} \hat{y}_{\alpha}(o) \hat{y}_{\sigma+1}(p) \hat{y}_{\sigma+1}(q)\right\} .
\end{aligned}
$$

The primed sums indicate the summation variables to be mutually different. Note that this ansatz reflects a particular choice of parameters for the general ansatz. Inserting the trial function into $F[g]$ yields the following functional:

$$
\begin{aligned}
D[g]= & \sum_{\alpha=1}^{\sigma}\left\{\frac { 2 d } { ( 2 d + 1 ) ^ { 2 } } f ( W _ { \alpha } ) W _ { \alpha } \sum _ { l = 0 } ^ { L } c _ { l } \left[\left(\frac{1}{L+1}\right.\right.\right. \\
& \left.+\left(a_{l}^{\alpha}-a_{l+1}^{\alpha}\right)-\frac{2 d}{(2 d+1)}\left(c_{l, l+1}^{\alpha}-c_{l+1, l}^{\alpha}\right)\right)^{2} \\
& +\frac{2 d}{(2 d+1)^{2}} \sum_{k^{\prime}}^{\prime \prime}\left(c_{0 l, k^{\prime}}^{\alpha}-c_{l+1, k^{\prime}}^{\alpha}\right. \\
& \left.-\frac{2 d}{(2 d+1)}\left(e_{l,\left(l+1, k^{\prime}\right)}^{\alpha}-e_{l+1,\left(l, k^{\prime}\right)}^{\alpha}\right)\right)^{2} \\
& +\sum_{\beta=1}^{\sigma} \frac{2 d}{(2 d+1)} f\left(W_{\beta}\right) \sum_{k}^{\prime \prime}\left(c_{k, l}^{\beta}-c_{k, l+1}^{\beta}\right)^{2} \\
& +\frac{4 d^{2}}{(2 d+1)^{4}} \sum_{p<q}^{\prime \prime}\left(e_{l, p, q}^{\alpha}-e_{l+1, p, q}^{\alpha}\right)^{2} \\
& \left.\left.+\sum_{\beta=1}^{\sigma} \frac{4 d^{2}}{(2 d+1)^{3}} f\left(W_{\beta}\right) \sum_{o, q}\left(e_{o,(l, q)}^{\beta}-e_{o,(l+1, q)}^{\beta}\right)^{2}\right]\right\} .
\end{aligned}
$$

The free parameters are the coefficients $a_{i}^{\alpha}, c_{k, k^{\prime}}^{\alpha}$, and $e_{o, p, q}^{\alpha}$ which are, generalizing results in Ref. [15], chosen as

$$
\begin{gathered}
a_{i+1}^{\alpha}=\sum_{l=0}^{i}\left(\frac{1}{L+1}-\frac{2 d}{(2 d+1)}\left(c_{l, l+1}^{\alpha}-c_{l+1, l}^{\alpha}\right),\right. \\
a_{0}^{\alpha}=0,
\end{gathered}
$$

$$
c_{i, j}^{\alpha}= \begin{cases}\frac{(2 d+1) C}{2 d}\left(1-g\left(\frac{i}{n}\right)\right) \frac{L+1-j}{(L+1)^{2}} & \text { for } i<j \\ -c_{L+1-i, L+1-j}^{\alpha} & \text { for } i>j,\end{cases}
$$

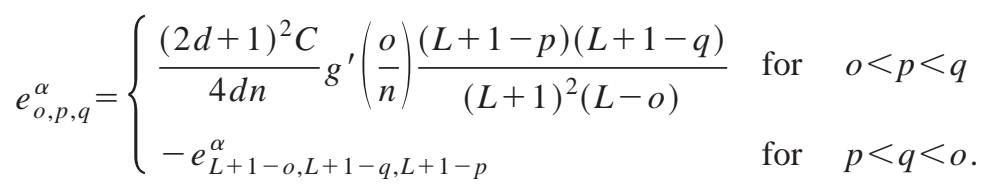

the real numbers with $g(x)=1$ for $x \leqslant 0$ which is decreasing exponentially fast for $x \rightarrow \infty$. This function has the property that

$$
\sum_{i=0}^{\infty}\left[g^{(r)}\left(\frac{i}{n}\right)\right]^{s}=O(n),
$$

where $r, s$ are integers and $n=(L+1)^{0.75}$. Given this choice, the following upper bound for $D_{L+1}^{\text {open }}$ is found:

$$
D_{L+1}^{o p e n} \leqslant \frac{1}{(2 d+1)} \frac{1}{(L+1)^{2}}\langle W\rangle
$$

where $\langle W\rangle$ is the disorder average.

This completes the proof that asymptotically $D \propto 1 / L^{2}$ in the presence of kinematic disorder.

\section{MC RESULTS}

In the case of the ordered RD model, the results for $D$ in the periodic case [14] coincide to leading order with the up- 


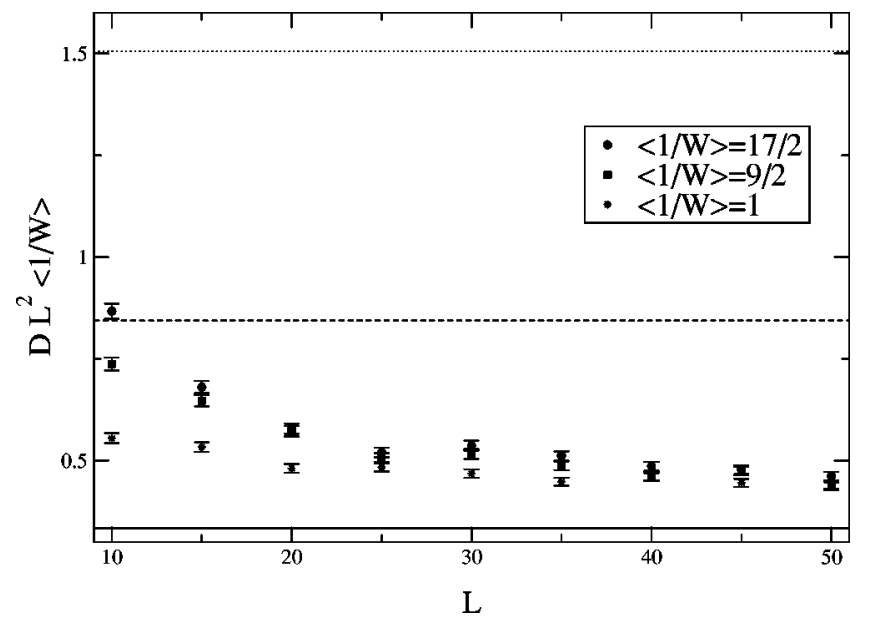

FIG. 3. $D(0) L^{2}\langle 1 / W\rangle$ vs $L$ for the cases as described in the text. The lower bound for all three cases is given by the solid line. The dotted line is the upper bound for the case with $\langle 1 / W\rangle=17 / 2$ and the dashed one the upper bound when $\langle 1 / W\rangle=9 / 2$. The case with $\langle 1 / W\rangle=1$ is the ordered case where both bounds coincide.

per bound for the open case [15]. In our model, we managed to give bounds on $D$ which both scale with $1 / L^{2}$ in the limit for long chains. This implies that kinematic disorder does not ruin the scaling relation of the standard reptation theory. Still, the numerical prefactors differ. We believe that it is the result for the lower bound, which correctly describes the diffusive behavior for long chains. This lower bound was obtained by a rigorous calculation, while the upper bound results from a variational treatment. Given that our conjecture is true and for long chains $D=1 /\left[L^{2}(2 d+1)\langle 1 / W\rangle\right]$, then for any choice of disorder distribution, $D L^{2}\langle 1 / W\rangle$ plotted against the chain length is constant. We performed MC simulations with different disorder distributions. The upper and lower bounds differ significantly for binary distributions. We compare in Fig. 3 the ordered case with $d=1$ (limit for $\left.D L^{2}\langle 1 / W\rangle=1 / 3\right)$ to the cases $W_{1}=1 / 16, W_{2}=1, f\left(W_{1}\right)$ $=f\left(W_{2}\right)=1 / 2,\langle 1 / W\rangle=17 / 2$ (upper bound for $D L^{2}\langle 1 / W\rangle$ is 1.505 , lower one 1/3) and $W_{1}=1 / 8, \quad W_{2}=1, f\left(W_{1}\right)$ $=f\left(W_{2}\right)=1 / 2,\langle 1 / W\rangle=9 / 2$ (upper bound for $D L^{2}\langle 1 / W\rangle$ is 0.844 , lower one $1 / 3)$. These results suggest that in the long chain length limit, $D=1 /\left[L^{2}(2 d+1)\langle 1 / W\rangle\right]$.

\section{DYNAMICS OF INTERNAL SEGMENTS}

The surprising result of the previous sections is the observation that the effect of kinematic disorder on the collective behavior of all connected polymer segments is (to leading order in system size) the same as on a simple pointlike object in the same disorder environment. In order to understand this observation, we now consider the dynamics of the internal segments in the hydrodynamic limit of the vanishing lattice spacing. For the local concentration of reptons, one obtains from the usual ordered repton model in this limit Rouse dynamics [13] restricted to motion inside the tube [5]. The boundary dynamics, i.e., the hopping into and out of the tube describe the entropic tensile force acting on the chain ends and keeping the polymer in its stretched equilibrium confor- mation. For understanding the dependendence of the diffusion coefficient on kinematic disorder, which affects mostly the bulk of the polymer chain, it is sufficient to focus on the hydrodynamic behavior of the bulk reptons. In order to study this limit, it is convenient to first investigate the associated zero-range process and then translate the result into the hydrodynamic limit of the exclusion process.

For the zero-range process described in Sec. III with $E$ $=0$, the average number of particles $\rho_{i}(t)$ at site $i$ obeys the exact time evolution equation given by

$$
\frac{\partial \rho_{i}(t)}{\partial t}=W_{i} z_{i-1}(t)+W_{i+1} z_{i+1}(t)-\left(W_{i}+W_{i+1}\right) z_{i}(t)
$$

where $z_{i}(t)$ is the probability that site $i$ is occupied at time $t$. It is known that in the steady state, the occupancy probability $z_{i}^{s s}$ is spatially uniform and can be related to the steady state particle density as [19]

$$
z_{i}^{s s}=\frac{\rho_{i}^{s s}}{1+\rho_{i}^{s s}} .
$$

It follows that the steady state density profile is uniform in spite of disorder.

To understand the dynamics in the hydrodynamic limit of vanishing lattice spacing, we expand Eq. (64) to second order in lattice constant and find

$$
\frac{\partial \rho(x, t)}{\partial t}=\frac{\partial}{\partial x}\left[W(x) \frac{\partial z(x, t)}{\partial x}\right] .
$$

At large enough time, the system is expected to be in local equilibrium so that we may assume the usual approximation for $z(x, t)-\rho(x, t)$ in the steady state to be valid: $z(x, t)$ $\approx \rho(x, t) /[1+\rho(x, t)]$, where Eq. (65) was used. We are interested in the density fluctuations about the steady state, $\Delta \rho(x, t)=\rho(x, t)-\rho^{s s}(x)$ where $\rho^{s s}(x)=\rho=K / M$. Retaining the lowest nonvanishing term in the expansion of the preceding equation in powers of $\Delta \rho(x, t)$, we obtain

$$
\frac{\partial \Delta \rho(x, t)}{\partial t}=\frac{1}{(1+\rho)^{2}} \frac{\partial}{\partial x}\left[W(x) \frac{\partial \Delta \rho(x, t)}{\partial x}\right] .
$$

The above equation describes a random walker in one dimension, diffusing in a random medium with bondsymmetric hopping rates $W(x)$. It can be shown that at large time and length scales, the random walker can be described by a single, effective diffusion constant $\mathcal{D}=1 /[(1$ $\left.+\rho)^{2}\langle 1 / W\rangle\right]$, provided $\langle 1 / W\rangle$ is finite [25]. Thus, we obtain the bulk diffusion constant to be given by $\mathcal{D}$ in the zerorange process.

Regarding the site as particle and mass as hole clusters, the above model maps onto symmetric exclusion process (SEP) with particlewise disorder. We want to calculate the bulk diffusion constant in the SEP picture using the above results for zero-range process. Since the steady state density profile is uniform in both the pictures, the average local density $n_{i}$ in the vicinity of the location of particle $i$ in SEP is 
related to that in the zero-range process as $n_{i}=1 /\left(1+\rho_{i}\right)$. Then, the density fluctuation about the steady state in the vicinity of particle $i$ is given by

$$
\Delta n_{i}=\frac{-1}{(1+\rho)^{2}} \Delta \rho_{i}+O\left((\Delta \rho)^{2}\right) .
$$

We note that the above is true only at large enough times, as was pointed out in a similar analysis for the tagged particle correlation function for SEP without disorder [26].

Using Eqs. (67) and (68), we find that

$$
\frac{\partial \Delta n(x, t)}{\partial t}=\frac{1}{(1+\rho)^{2}} \frac{\partial}{\partial x}\left[W(x) \frac{\partial \Delta n(x, t)}{\partial x}\right] .
$$

Further, note that, $x$ is the space index in the zero-range process, while it labels the particles in the SEP. The space coordinate $y$ in symmetric exclusion process is related to $x$ as

$$
y \approx \int^{x} d x^{\prime} \rho\left(x^{\prime}\right)+x
$$

which gives

$$
\frac{\partial}{\partial x}=(1+\rho) \frac{\partial}{\partial y}+O(\Delta n) .
$$

Thus, the particle density fluctuations in the SEP obey

$$
\frac{\partial \Delta n(y, t)}{\partial t}=\frac{\partial}{\partial y}\left[W(y) \frac{\partial \Delta n(y, t)}{\partial y}\right] .
$$

Using the random walker analogy, we obtain the effective diffusion constant $\overline{\mathcal{D}}$ at large times to be equal to $1 /\langle 1 / W\rangle$ in the symmetric exclusion process.

This calculation shows that the internal segments of the polymer chain perform Rouse-type dynamics also in the presence of kinematic disorder, but with a disorderdependent diffusion coefficient. This explains the occurrence of the same correction to the diffusion coefficient for the long time behavior of the polymer chain as a whole.

\section{CONCLUSIONS}

It is the aim of this paper to disentangle the effects of the various types of disorder, which one may expect to have significant impact on the dynamics of systems of entangled flexible polymers. We have focused on kinematic disorder which leaves the equilibrium conformation unchanged compared to a hypothetical ordered entanglement network (which could, in principle, be manufactured artificially by placing a single polymer in an ordered array of obstacles on a surface). For the periodic RD model with kinematic disorder, we computed the drift velocity in the presence of an external field in the linear response regime. Knowing the diffusion constant for the model with open boundaries yields via the Einstein relation the drift velocity in this case. We have proved (rigorously in terms of the RD model for reptation) that the asymptotic length scaling of the diffusion coefficient of the polymer chain remains as predicted by the standard reptation theory. By studying the hydrodynamic limit, we have shown that the individual polymer segments inside the tube perform Rouse dynamics in a disordered environment, which corresponds to a system of local random barriers. Therefore, the amplitude of the diffusion coefficient becomes dependent on the disorder in the same way a single particle in a random barrier system.

\section{ACKNOWLEDGMENTS}

K.J. wishes to thank the Forschungszentrum Jülich for kind hospitality. R.D.W. thanks Deutsche Forschungsgemeinschaft for financial support.
[1] P.G. de Gennes, Scaling Concepts in Polymer Physics (Cornell University Press, Ithaca, 1979).

[2] T.P. Lodge, Phys. Rev. Lett. 83, 3218 (1999).

[3] T.P. Lodge and E.D. von Meerwall, Macromolecules 33, 1747 (2000).

[4] J.D. Ferry, Viscoelastic Properties of Polymers (Wiley, New York, 1980).

[5] M. Doi, Introduction to Polymer Physics (Oxford University Press, Oxford, 1997).

[6] E. Carlon, A. Drzewinski, and J.M.J. van Leeuwen, Phys. Rev. E 64, 010801(R) (2001).

[7] M. Paessens and G.M. Schütz, Phys. Rev. E 66, 021806 (2002).

[8] M. Rubinstein, Phys. Rev. Lett. 59, 1946 (1987).

[9] J.M. Deutsch and T.L. Madden, J. Chem. Phys. 91, 3252 (1989).

[10] T.A.J. Duke, Phys. Rev. Lett. 62, 2877 (1989).

[11] T.A.J. Duke, J. Chem. Phys. 93, 9049 (1990).
[12] G.T. Barkema, C. Caron, and J.F. Marko, Biopolymers 38, 665 (1996).

[13] G.M. Schütz, Europhys. Lett. 48, 623 (1999).

[14] J.M.J. van Leeuwen and A. Kooiman, Physica A 184, 79 (1992).

[15] M. Prähofer and H. Spohn, Physica A 233, 191 (1996).

[16] A. Baumgärtner and M. Muthukumar, in Advances in Chemical Physics, edited by I. Prigogine and S.A. Rice (Wiley, New York, 1996), Vol. XCIV.

[17] A.E. Likhtman, S.T. Milner, and T.C.B. McLeish, Phys. Rev. Lett. 85, 4550 (2000).

[18] L. Schäfer, A. Baumgärtner, and U. Ebert, Eur. Phys. J. B 10, 105 (1999).

[19] R.D. Willmann, J. Chem. Phys. 116, 2688 (2002).

[20] G.M. Schütz, in Phase Transitions and Critical Phenomena, edited by C. Domb and J.L. Lebowitz (Academic, London, 2000), Vol. 19.

[21] M. Prähofer, Diploma thesis, LMU Munich, 1994. 
[22] I. Benjamini, P.A. Ferrari, and C. Landim, Stochastic Proc. Appl. 61, 181 (1996).

[23] B. Derrida, J. Stat. Phys. 31, 433 (1983).

[24] H. Spohn, Large Scale Dynamics of Interacting Particles (Springer, Heidelberg, 1991).
[25] K.W. Kehr, K. Mussawisade, G.M. Schütz, and T. Wichmann, in Diffusion in Condensed Matter, edited by P. Heitjans and J. Kräger (Springer, Berlin, 2003).

[26] S. Alexander and P. Pincus, Phys. Rev. B 18, 2011 (1978). 